\title{
A Slow-I-Fast-P Architecture for Compressed Video Action Recognition
}

\author{
Jiapeng Li \\ lijiapeng@stu.xjtu.edu.cn \\ Xi'an Jiaotong University, Xi'an, China \\ Yongchi Zhang \\ yongchizh@stu.xjtu.edu.cn \\ Xi'an Jiaotong University, Xi'an, China
}

\author{
Ping Wei* \\ pingwei@xjtu.edu.cn \\ Xi'an Jiaotong University, Xi'an, China \\ Nanning Zheng \\ nnzheng@mail.xjtu.edu.cn \\ Xi'an Jiaotong University, Xi'an, China
}

\begin{abstract}
Compressed video action recognition has drawn growing attention for the storage and processing advantages of compressed videos over original raw videos. While the past few years have witnessed remarkable progress in this problem, most existing approaches rely on RGB frames from raw videos and require multi-step training. In this paper, we propose a novel Slow-I-Fast-P (SIFP) neural network model for compressed video action recognition. It consists of the slow I pathway receiving a sparse sampling I-frame clip and the fast $\mathrm{P}$ pathway receiving a dense sampling pseudo optical flow clip. An unsupervised estimation method and a new loss function are designed to generate pseudo optical flows in compressed videos. Our model eliminates the dependence on the traditional optical flows calculated from raw videos. The model is trained in an endto-end way. The proposed method is evaluated on the challenging HMDB51 and UCF101 datasets. The extensive comparison results and ablation studies demonstrate the effectiveness and strength of the proposed method.
\end{abstract}

\section{CCS CONCEPTS}

- Computing methodologies $\rightarrow$ Activity recognition and understanding; Computer vision representations.

\section{KEYWORDS}

action recognition; compressed video; neural networks

\section{ACM Reference Format:}

Jiapeng Li, Ping Wei, Yongchi Zhang, and Nanning Zheng. 2020. A SlowI-Fast-P Architecture for Compressed Video Action Recognition. In Proceedings of the 28th ACM International Conference on Multimedia (MM '20), October 12-16, 2020, Seattle, WA, USA. ACM, New York, NY, USA, 9 pages. https://doi.org/10.1145/3394171.3413641

\section{INTRODUCTION}

With the proliferation of huge amounts of videos from social media and mobile devices, traditional video understanding frameworks

\footnotetext{
*Ping Wei is the corresponding author.

Permission to make digital or hard copies of part or all of this work for personal or classroom use is granted without fee provided that copies are not made or distributed for profit or commercial advantage and that copies bear this notice and the full citation on the first page. Copyrights for third-party components of this work must be honored.

For all other uses, contact the owner/author(s).

MM '20, October 12-16, 2020, Seattle, WA, USA

(C) 2020 Copyright held by the owner/author(s).

ACM ISBN 978-1-4503-7988-5/20/10.

https://doi.org/10.1145/3394171.3413641
}

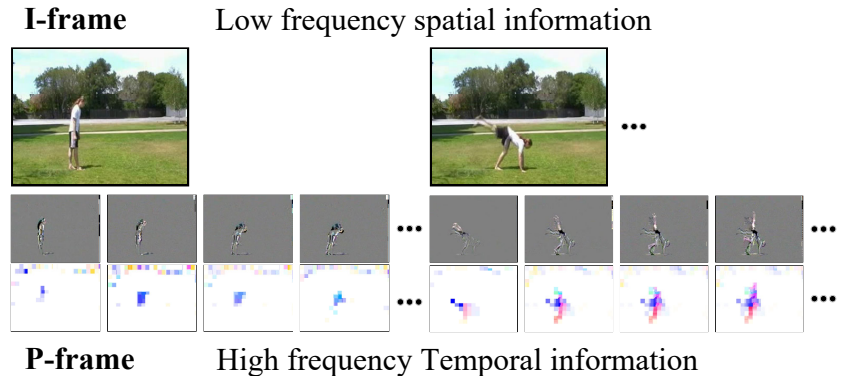

Figure 1: Illustration of 'slow' and 'fast' information in compressed videos. Compressed video contains low sampling rate $I$-frames and high sampling rate $P$-frames. The $I$-frames describe spatial information at low frequency while the MV (motion vector) in P-frames can characterize temporal information at high frequency.

using raw RGB videos have encountered great storage and processing obstacles. Most traditional architectures utilize RGB frames or optical flows for video action recognition [2, 33, 34]. However, it takes huge storage and time resource to decode compressed videos into raw RGB videos [50]. And also it often takes a great deal of time to calculate optical flows through RGB images and a large space to store the calculated optical flows [32].

To overcome these challenges, many studies $[50,53,54]$ over the recent years have moved to action recognition with compressed videos encoded by MPEG-4 [22] or other standards. While these studies have achieved great progress in compressed video action recognition, there is still a large gap in performance compared to the frameworks using raw RGB videos. In order to improve the performance, some methods introduce optical flows into compressed video features as extra information [32, 50]. However, most existing methods have to be trained in a multi-step manner rather than an end-to-end way. For example, CoViAR [50] and DMC-Net [32] utilize multiple streams, each of which needs to be trained separately. Such strategy lowers the model efficiency and limits the performance improvement. Second, these methods are not 'pure' compressed video action recognition. Although they seek to recognize actions with compressed videos instead of RGB frames in raw videos, they have to use optical flows which are calculated by RGB frames in raw videos $[15,36,49,52]$. 
Recently, a SlowFast model [8] has been proposed for recognizing human actions in raw RGB videos. The SlowFast framework is enlightened by the primate visual system [5, 12, 16, 24, 42] and contains two pathways - slow pathway and fast pathway. The slow pathway takes as input RGB images with low frame rate and the fast pathway addresses RGB images with high frame rate. With this mechanism, the spatial semantic information and the fast changing motion are both captured for action analysis [8].

In fact, compressed videos naturally contain 'slow' and 'fast' information. A compressed video consists of a series of GOPs (group of pictures), each of which contains an I-frame as a reference and multiple consecutive P-frames [50]. As shown in Fig. 1, the I-frames describe spatial information at low frequency while the MV (motion vector) in P-frames can characterize temporal information at high frequency.

Inspired by the SlowFast framework [8], in this paper we propose a Slow-I-Fast-P (SIFP) network for compressed video action recognition. It mainly consists of two interconnected pathways, the slow I pathway taking a sparse sampling I-frame clip as input, and the fast $\mathrm{P}$ pathway taking a dense sampling pseudo optical flow clip generated by P-frames as input. To take the advantage of optical flow features but avoid using RGB frames from raw videos, we propose a generator which aims to produce pseudo optical flows with residuals (Res) and motion vectors (MV) decomposed from P-frames. A new unsupervised loss function is designed to train the generation of the pseudo optical flows in compressed videos. In this way, our method gets rid of the dependence on the RGB images and traditional optical flow features. The network is trained in an end-to-end manner. We evaluate the proposed method on the UCF101 [35] and HMDB51 [21] datasets. Extensive experiments on the datasets prove the strength of our method.

\section{RELATED WORK}

Video Action Recognition. Human action recognition is a challenging problem and can be applied in many applications $[2,8,33$, $41,47,48]$. Two-stream models [20, 33, 34, 44] and 3D convolution models $[2,29,38,40,41,51]$ are two representative streams of neural network methods for action recognition. Two-stream methods utilize optical flow features and RGB image features as two streams for action recognition. The computation of optical flow is often implemented offline and very time-consuming. And therefore most two-stream models are difficult to be trained in an end-to-end way. $3 \mathrm{D}$ convolution methods could only rely on RGB information and achieve impressive performance in end-to-end ways. Some 3D convolution methods $[2,41,51]$ also integrate optical flow information to improve performance but need to be trained in multiple steps.

The SlowFast model [8] is an inspiring architecture, which exploits the slow and fast information in videos for action recognition. Our Slow-I-Fast-P (SIFP) model is inspired by the SlowFast model [8]. But it is different from SlowFast in three aspects. First, SlowFast takes as input raw RGB frames at different frame rate and our SIFP takes I-frames and P-frames as inputs. Second, our SIFP architecture embraces a generator to produce pseudo optical flows. Third, an unsupervised loss function is proposed in our SIFP to guide the generation of pseudo optical flows.
Compressed Video Action Recognition. Action recognition with compressed videos has drawn increasing attention in recent years. In the pioneering studies [53, 54], motion vectors are used to replace optical flow features. However in these methods compressed videos and raw videos are simultaneously used for prediction. The CoViAR model [50] utilizes three streams of 2D convolutional networks to extract I-frame, motion vector (MV) and residual (Res) features, respectively. This model needs to be trained in three steps separately. The DMC-Net model [32] proposes a generator and combines MV with Res as a new stream to generate motion cues. However it uses optical flow features to supervise the generation process.

Inspired by these studies, we seek to integrate the generation of pseudo optical flows into an end-to-end framework. Furthermore, we design an unsupervised loss function to regulate the generation.

Optical Flow Estimation. Optical flows are important features in videos to characterize motion information [15]. With deep neural networks, many methods have achieved high precision to estimate optical flows [6, 13, 17, 30,37] on some challenging benchmarks $[1,27]$, such as FlowNet [6], SPyNet [30] and PWC-Net [37]. Many studies apply optical flow estimation to action recognition and have achieved impressive results [23, 28, 55]. DMC-Net [32] utilizes motion cues to replace optical flows for action recognition, which is proved to be reasonable and helpful. However, Sevilla-Lara et al. [31] showed that the accuracy of optical flow estimation and the performance of action recognition are not strongly correlated. What is really relevant to action recognition is the general motion information contained in optical flows. This suggests other possibilities to characterize motion information for action recognition.

Due to the huge calculation cost of optical flows, unsupervised optical flow estimation is becoming growingly important $[18,26$, 46]. Unsupervised loss functions allow the model to be trained without optical flow benchmarks. Among these unsupervised loss functions, the photometric loss and the smoothness loss are most widely used in unsupervised methods $[18,26,46]$. Based on the assumption of photometric consistency, the photometric loss function calculates the final loss with the estimated optical flows and two RGB frames before and after the current motion. However, there are no continuous RGB frames in compressed videos. We improve the original photometric loss and propose a residual photometric loss for compressed videos.

\section{APPROACH}

In this section, we introduce the proposed Slow-I-Fast-P (SIFP) framework in detail. We briefly introduce the data format of compressed videos and then elaborate on the overall SIFP architecture. Finally, we describe the residual photometric loss function.

\subsection{Data Format of Compressed Video}

Following the CoViAR model [50] and the DMC-Net model [32], we use MPEG-4 Part 2 [22] to encode action videos. The compressed video via MPEG-4 is usually packed into multiple GOPs (group of pictures). Each GOP contains 12 frames, including an I-frame and 11 consecutive P-frames. Each P-frame can be decomposed into residual and motion vector. Therefore, three data modalities can be obtained from the compressed video: 


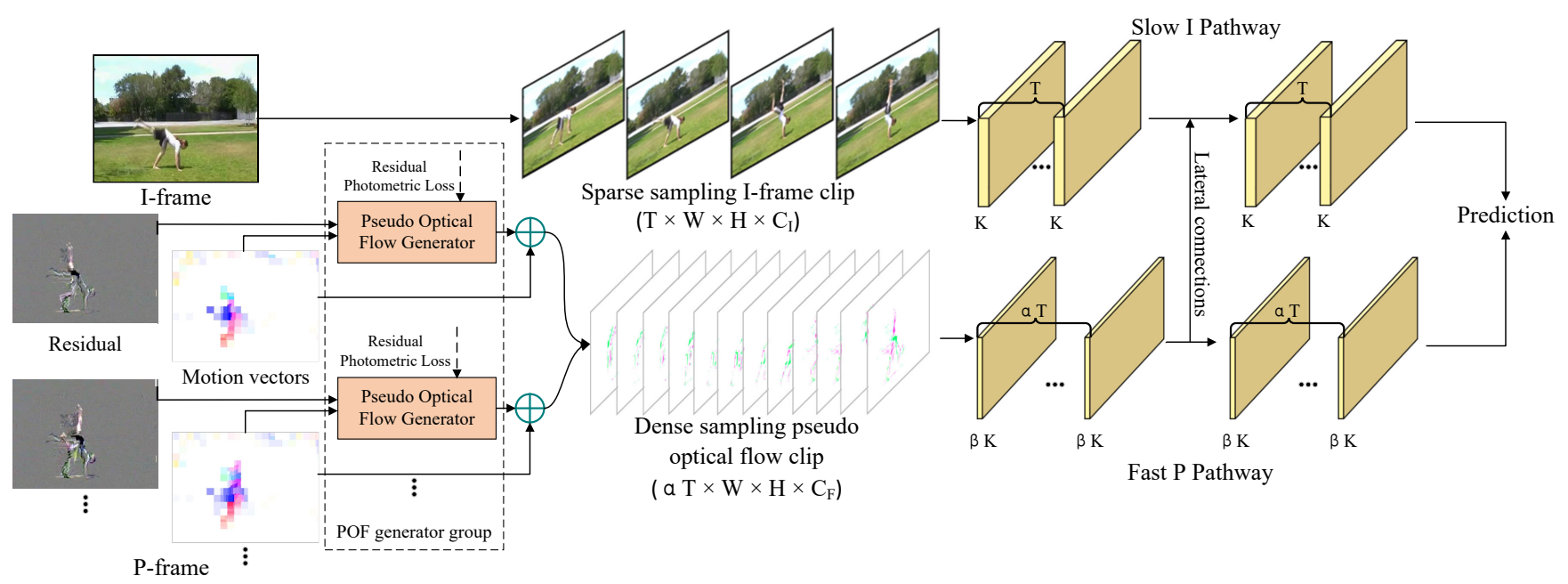

Figure 2: Illustration of the SIFP model structure. SIFP contains two pathways and a serials of POF generators. The slow I pathway takes as input a sparse I-frame clip, and the fast $P$ pathway receives a dense pseudo optical flow clip generated by the serials of POF generators.

- I-frame represents the reference RGB frame of each GOP.

- Motion vector (MV) describes the displacement of each $16 \times$ 16 macroblock relative to the reference I-frame or the last $\mathrm{P}$-frame [32]. It represents the approximate movement of the target.

- Residual (Res) maintains the RGB differences between a real frame and its reconstructed frame calculated by MV after motion compensation [32].

\subsection{Slow-I-Fast-P Networks}

Our proposed Slow-I-Fast-P (SIFP) framework consists of three major parts: slow I pathway, fast $\mathrm{P}$ pathway, and a series of $2 \mathrm{D}$ pseudo optical flow generators called pseudo optical flow (POF) generator group, as shown in Fig. 2. The slow I pathway takes a sparse sampling I-frame clip as input, and the fast $\mathrm{P}$ pathway takes a dense sampling pseudo optical flow clip generated by P-frames as input. The POF generator group receives a P-frame clip and outputs the pseudo optical flows to characterize motion information. The slow I pathway and the fast $\mathrm{P}$ pathway are inspired by the SlowFast model [8] and adopt the same structure with SlowFast. The input structure of our SIFP is different from the SlowFast which addresses raw RGB frames.

The whole structure of the network is similar to the two-stream framework [33, 34] which contains the RGB stream and the optical flow stream. The slow I pathway is similar to the RGB stream while the fast $\mathrm{P}$ pathway is similar to the optical flow stream. Different from the traditional two-stream frameworks, there are connections between the two pathways, which enable our model to be trained in an end-to-end way.

Slow I pathway and Fast P pathway. As shown in Fig. 2, the slow I pathway takes a sparse I-frame clip as input and the shape of the input clip is $T \times W \times H \times C_{I}$, where the parameters are the temporal length of the clip, the width, height, and channel of the I-frame, respectively. The fast $\mathrm{P}$ pathway receives a dense pseudo optical flow clip generated by $\mathrm{P}$-frames as input, and the shape of the input clip is $\alpha T \times W \times H \times C_{F}$, where $\alpha$ is a temporal sampling parameter as defined in the SlowFast model [8].

With the inputs, the two pathways are both composed of cascaded 3D ResNet blocks $[39,45]$ and adopt the same settings as in SlowFast [8]. In the slow I pathway, for a convolutional structure in a ResNet block, suppose the temporal length of the feature map is $T$ and the kernel number is $K$. For the corresponding structure in the fast $\mathrm{P}$ pathway, the temporal length of the feature map is $\alpha T$ and the kernel number is $\beta K$, where $\alpha>1$ and $\beta<1$ are the temporal sampling parameter and the channel sampling parameter [8], respectively. According to SlowFast [8], with such sampling strategy, the slow I pathway would have low sampling frequency and high channel capacity. On the contrary, the fast $\mathrm{P}$ pathway would have high sampling frequency and low channel capacity. Thus, it is natural and feasible to process the compressed videos because the structure of compressed video contains sparse I-frames interspersed with dense P-frames.

We instantiate the two pathways by taking ResNet50 [14] as the backbone, and set $\alpha=4, \beta=\frac{1}{8}$. Table 1 shows the details of the instantiation, where $S$ is the spatial size of the output feature map.

Pseudo optical flow (POF) generator. Pseudo optical flow (POF) generator group takes a high-frequency P-frame clip as input, and the output is a pseudo optical flow clip keeping the same temporal frequency. Fig. 3 shows the structure of a single pseudo optical flow generator. A pair of Res and MV are input to the singel POF generator and the output is the corresponding pseudo optical flow.

For the internal structure of the generator, we adopt the same design and setting as in DMC-Net [32]. It consists of six densely connected convolution layers. Except for the last layer, each convolution layer uses Leaky ReLU [25] activation function with the negative slope 0.1 . 
Table 1: Instantiation of the slow I pathway and the fast $P$ pathway.

\begin{tabular}{|c|c|c|c|c|c|}
\hline Stage & \multicolumn{2}{|c|}{ Slow I pathway } & \multicolumn{2}{|c|}{ Fast P pathway } & Output $T \times S^{2}$ \\
\hline \multirow{2}{*}{ input } & \multirow{2}{*}{\multicolumn{2}{|c|}{ - }} & & Slow : $8 \times 224^{2}$ \\
\hline & & & & & Fast : $32 \times 224^{2}$ \\
\hline \multirow{2}{*}{$\operatorname{conv}_{1}$} & \multirow{2}{*}{\multicolumn{2}{|c|}{$\begin{array}{c}1 \times 7^{2}, 64 \\
\text { stride } 1,2^{2}\end{array}$}} & \multirow{2}{*}{\multicolumn{2}{|c|}{$\begin{array}{c}5 \times 7^{2}, 8 \\
\text { stride } 1,2^{2}\end{array}$}} & Slow : $8 \times 112^{2}$ \\
\hline & & & & & Fast : $32 \times 112^{2}$ \\
\hline \multirow{2}{*}{ pool $_{1}$} & \multirow{2}{*}{\multicolumn{2}{|c|}{$\begin{array}{l}1 \times 3^{2}, \max \\
\text { stride } 1,2^{2}\end{array}$}} & \multirow{2}{*}{\multicolumn{2}{|c|}{$\begin{array}{l}1 \times 3^{2}, \max \\
\text { stride } 1,2^{2}\end{array}$}} & Slow : $8 \times 56^{2}$ \\
\hline & & & & & Fast : $32 \times 56^{2}$ \\
\hline \multirow{3}{*}{$\mathrm{res}_{2}$} & \multirow{3}{*}[\begin{array}{c}{1\times1^{2},64}\\
{1\times3^{2},64}\\
{1\times1^{2},256}\end{array}]{} & \multirow{3}{*}{$\times 3$} & \multirow{3}{*}[\begin{array}{c}{3\times1^{2},8}\\
{1\times3^{2},8}\\
{1\times1^{2},32}\end{array}]{} & \multirow{3}{*}{$\times 3$} & \\
\hline & & & & & Slow : $8 \times 56^{2}$ \\
\hline & & & & & Fast : $32 \times 56^{2}$ \\
\hline \multirow{3}{*}{$\mathrm{res}_{3}$} & \multirow{3}{*}[\begin{array}{l}{1\times1^{2},128}\\
{1\times3^{2},128}\\
{1\times1^{2},512}\end{array}]{} & \multirow{3}{*}{$\times 4$} & \multirow{3}{*}{$\left.\begin{array}{l}3 \times 1^{2}, 16 \\
1 \times 3^{2}, 16 \\
1 \times 1^{2}, 64\end{array}\right]$} & \multirow{3}{*}{$\times 4$} & Slow $\cdot 8 \times 28^{2}$ \\
\hline & & & & & S10W : $8 \times 28$ \\
\hline & & & & & Fast : $32 \times 28^{2}$ \\
\hline \multirow{3}{*}{$\mathrm{res}_{4}$} & \multirow{3}{*}[\begin{array}{c}{3\times1^{2},256}\\
{1\times3^{2},256}\\
{1\times1^{2},1024}\end{array}]{} & \multirow{3}{*}{$\times 6$} & \multirow{3}{*}[\begin{array}{c}{3\times1^{2},32}\\
{1\times3^{2},32}\\
{1\times1^{2},128}\end{array}]{} & \multirow{3}{*}{$\times 6$} & \\
\hline & & & & & Slow : $8 \times 14^{2}$ \\
\hline & & & & & Fast : 3 \\
\hline \multirow{3}{*}{$\operatorname{res}_{5}$} & $3 \times 1^{2}, 512$ & \multirow{3}{*}{$\times 3$} & \multirow{3}{*}[\begin{array}{c}{3\times1^{2},64}\\
{1\times3^{2},64}\\
{1\times1^{2},256}\end{array}]{} & \multirow{3}{*}{$\times 3$} & \\
\hline & $1 \times 3^{2}, 512$ & & & & Fiow . $0 x$ \\
\hline & {$\left[1 \times 1^{2}, 2048\right.$} & & & & \\
\hline
\end{tabular}

As in DMC-Net [32], MV can be considered as a low resolution optical flow. MV is usually very rough and serrated at the edge of the image because it is based on simple block matching [32]. The generator can optimize the MV, smooth its edge, and eliminate the noise. Thus a MV shortcut connection is used in this structure [32], as shown in Fig. 3.

Res is calculated as the RGB differences between the original frames. We observe that Res is often aligned with the boundary of the moving object and therefore it may play a guidance role in MV smoothing and noise elimination. According to this observation, we not only adopt Res and MV as inputs, but also design a residual photometric loss based on Res as shown in Fig. 3. Our proposed residual photometric loss is improved by the classical unsupervised optical flow estimation loss function. Further discussion will be presented in Section 3.3.

Lateral connections. In previous methods, lateral connection is often used to fuse the two-stream networks $[9,10]$. Following SlowFast [8], we also adopt lateral connections to fuse fast $\mathrm{P}$ pathway and slow I pathway in our architecture for joint training. We use the T-conv connection [8] as the lateral connections. The fusion direction is fast-to-slow and the fusion mode we use is concatenation.

\subsection{Residual Photometric Loss}

The photometric consistency hypothesis indicates that the photometric quantities in two consecutive RGB frames are the same [18]. Based on the photometric consistency hypothesis, the traditional unsupervised photometric loss function is proposed for optical flow

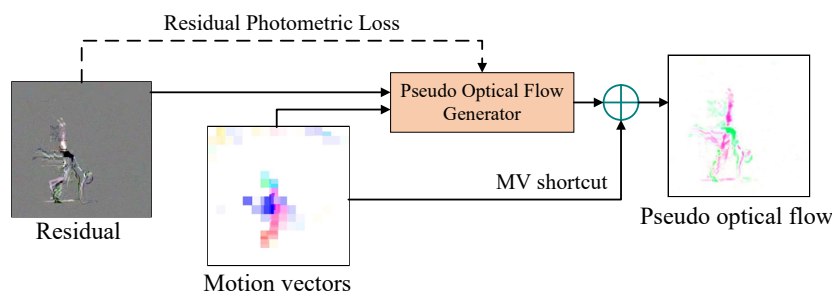

Figure 3: Illustration of the POF generator. The POF generator takes both residual and motion vector as inputs, and generates the pseudo optical flow under the regulation of the residual photometric loss.

estimation [18, 26, 46]. It has two sorts of definition forms: one is defined in the form of photometry, and the other is the image gradient. However the traditional photometric loss function cannot be directly applied to compressed videos. To calculate the traditional photometric loss, two successive RGB frames and the forward optical flow are required. However in compressed videos we only have two residuals rather than the two RGB frames.

We observe that there is a similarity between image gradients and residuals. Residuals are generated by motions which are often obviously reflected in the movement of object boundaries. Calculating image gradients can extract edges of objects. Therefore, residuals and gradients are usually consistent in appearance, which both reflect the boundary information of objects. This observation has also been noticed in the previous work [32]. In addition, the work [31] also found that the performance of action recognition and the accuracy of optical flow estimation are not necessarily related. What really related is the accuracy of optical flow at the edge and small displacement. Therefore, we consider to use residual to replace gradient and design a new residual photometric loss.

Following CoViAR [50], we employ the accumulated Res and $\mathrm{MV}$, where every previous frame of $\mathrm{P}$-frame is the I-frame of the current GOP. Suppose the I-frame of current GOP is $I_{1}$, and the current P-frame is $I_{2}$. The optical flow from the first frame $I_{1}$ to the second frame $I_{2}$ is set to be $F_{12}\left(F_{12}^{x}\right.$ in X direction, $F_{12}^{y}$ in $\mathrm{Y}$ direction), and the reverse optical flow is $F_{21}$.

Similar to the form of the traditional photometric loss [46], we define the residual photometric loss as:

$$
L_{R P}=\sum_{x, y} \Psi\left(\nabla \hat{I_{2}}(x, y)-\operatorname{Res}_{2}(x, y)\right)
$$

where $\operatorname{Res}_{2}(x, y)$ is the residual of the current P-frame, and $\Psi(s)$ is the charbonier penalty function to mitigate the effects of outliers, which is defined as $\sqrt{s^{2}+0.001^{2}}[26] . \hat{I}_{2}(x, y)$ is the warped $I_{2}$, which is calculated by I-frame $I_{1}$ and the reverse optical flow $F_{21}$ [46].

Based on the photometric consistency hypothesis, every point $x_{2}, y_{2}$ on $\hat{I_{2}}$ can be calculated as:

$$
\begin{aligned}
& x_{2}=x_{1}-F_{21}^{x}\left(x_{2}, y_{2}\right) \\
& y_{2}=y_{1}-F_{21}^{y}\left(x_{2}, y_{2}\right)
\end{aligned}
$$

where $\left(x_{1}, y_{1}\right)$ is a point on the I-frame $I_{1}$. Obstacles often results in $F_{21} \neq-F_{12}$. We add a convolution layer to learn the relationship 
between $F_{12}$ and $F_{21}$ for the calculation of reverse optical flows $F_{21}$ :

$$
F_{21}=W \times F_{12}+b
$$

where $W$ is the weight parameter in the convolution layer and $b$ is the bias. For specific implementation, we use a $3 \times 3$ convolution kernel without activation function.

Thus, $\hat{I}_{2}(x, y)$ is calculated as:

$$
\begin{aligned}
& x_{2}=x_{1}-\left[W \times F_{12}^{x}\left(x_{1}, y_{1}\right)+b\right] \\
& y_{2}=y_{1}-\left[W \times F_{12}^{y}\left(x_{1}, y_{1}\right)+b\right]
\end{aligned}
$$

The whole loss function of the network consists of two parts: the classification loss $L_{C L S}$ and the unsupervised residual photometric loss $L_{R P}$. The total loss $L$ of the network is defined as:

$$
L=L_{C L S}+\lambda L_{R P}
$$

The parameter $\lambda$ in Eq. (5) is a penalty factor to balance the classification loss and the residual photometric loss. If the penalty factor $\lambda$ is too large in the initial stage, the pseudo optical flows cannot be generated well. Therefore, the penalty factor $\lambda$ is designed to be gradually increased as:

$$
\lambda= \begin{cases}\frac{e}{\lambda_{e}} & e \leq E \\ \frac{E}{\lambda_{e}} & E<e\end{cases}
$$

where $e$ is the number of current epochs, and $\lambda_{e}>0$ is a constant. $E$ is used to control the growth of the $\lambda$. In the initial stage of training, $\lambda$ grows with the number of current epoch $e$ when the current epoch number $e$ is less than $E$. In the fluctuation phase, $\lambda$ remains fixed when the current epoch number $e$ is bigger than $E$. In our experiment we set $E$ as 14 empirically.

\section{EXPERIMENTS}

In this section, we describe the details of experiment settings, compare our model with other state-of-the-art methods, and conduct ablation studies with visualization.

\subsection{Datasets}

We perform extensive experiments on UCF101 [35] and HMDB51 [21] datasets, which are widely used in compressed video action recognition. The UCF101 contains 13320 videos from YouTube, and each source video usually corresponds to one action. Therefore the UCF101 is usually regarded as a scene-related dataset [19]. The HMDB51 contains 6766 videos, and most of them are cropped from movies, which means a source video in this dataset usually corresponds to multiple actions. Thus HMDB51 is a temporal-related dataset [19]. Both the two datasets are challenging in the field of video action recognition.

\subsection{Implementation Details}

Data preprocessing. We adopt the same data format as in CoViAR [50]. Each compressed video consists of a series of GOPs (group of pictures) with 12 frames. If there is only one I-frame in the slow I pathway, it would be too short and not consistent with the design of low frequency sampling. Furthermore, since 11 is a prime number, it is difficult to make the slow I pathway and fast $\mathrm{P}$ pathway sampled at the same intervals. Thus in the experiment, we repeat the first
Table 2: Accuracy comparison with the compressed video based methods (\%).

\begin{tabular}{lccc}
\hline Method & Optical flow & HMDB51 & UCF101 \\
\hline EMV-CNN [53] & No & 51.2 & 86.4 \\
DTMV-CNN [54] & No & 55.3 & 87.5 \\
CoViAR [50] & No & 59.1 & 90.4 \\
CoViAR + Flow [50] & Yes & 70.2 & 94.9 \\
PWC-Net + CoViAR [37] & Train & 62.2 & 90.6 \\
DMC-Net (ResNet-18) [32] & Train & 62.8 & 90.9 \\
DMC-Net (I3D) [32] & Train & 71.8 & 92.3 \\
\hline SIFP-Net (ours) & No & 72.3 & 94.0 \\
\hline
\end{tabular}

$\mathrm{P}$-frame in each GOP, increasing the basic ratio of P-frame over I-frame as $12: 1$.

Training. We pre-train the model on Kinetics400 [2], and finetune it on the two datasets. Four 2080Ti GPUs are used to train the whole model, with the batch size of 12 and the initial learning rate of 0.001 . We train 30 epochs on each dataset. For UCF101, the penalty function $\lambda_{e}$ is empirically set as 500 , while it is set as 50 on HMDB51. All the video frames are cut to $256 \times 224$, which is then cropped to $224 \times 224$ at random. We randomly flip all inputs for data augmentation in the training stage. For very short videos, we conduct loop playback to collect clips. These settings are similar to other 3D CNN methods [8, 41].

Test. We employ the general action recognition evaluation metric (video accuracy) as in 3D-CNN $[8,41]$ during test. Each video is divided into 10 clips evenly, and loop playback is set for short videos. For each $256 \times 224$ frame, we use three spatial sampling schemes, including cropping the left, middle, and right side of the frame to obtain a $224 \times 224$ window. Finally, 30 clips are collected in total. For all clips, the sum of their logits is calculated to get the classification result with the highest score.

\subsection{Comparison with SOTA}

In this part, we compare our method with the previous models. The comparison includes compressed video based methods and raw video based method.

Comparison with compressed video based methods. We compare our method with other methods which utilize compressed videos for action recognition. The comparison results are shown in Table 2. This table also indicates whether a method uses optical flows or not.

Compared with the methods which do not use optical flows such as EMV-CNN [53], DTMV-CNN [54], and CoViAR [50], our SIFP method outperforms them by a large margin on both the HMDB51 and UCF101 datasets. Some approaches use optical flow features only in training, such as PWC-Net + CoViAR [37], DMCNet (ResNet-18) [32], and DMC-Net (I3D) [32], and our method still outperforms them on the two datasets. These results prove the effectiveness and strength of our method. For the method CoViAR + Flow [50] which utilizes optical flows, our method performs better than it on the HMDB51 dataset but is slightly inferior on UCF101 dataset. The results also show that our scheme using unsupervised 
Table 3: Accuracy comparison with other raw video based methods (\%).

\begin{tabular}{lccc}
\hline Method & Optical flow & HMDB51 & UCF101 \\
\hline Motion representation & & & \\
ActionFlowNet (2f) [28] & Train & 43.6 & 71.0 \\
ActionFlowNet [28] & Train & 56.4 & 83.9 \\
TVNet [7] & Train & 71.0 & 94.5 \\
MARS(16f) [4] & Train & 72.3 & 94.6 \\
\hline 2D two-stream & & & \\
Two-Stream [33] & Yes & 43.6 & 71.0 \\
Two-Stream fusion [11] & Yes & 56.4 & 83.9 \\
ST-ResNet [9] & Yes & 66.4 & 93.4 \\
TSN [44] & Yes & 68.5 & 94.0 \\
\hline 3D convolution & & & \\
C3D [38] & No & 51.6 & 82.3 \\
Res3D [39] & No & 54.9 & 85.8 \\
P3D [29] & No & - & 88.6 \\
ARTNet [43] & No & 70.9 & 94.3 \\
ECO [56] & No & 72.4 & 94.8 \\
MF-Net [3] & No & 74.6 & 96.0 \\
I3D [2] & No & 74.8 & 95.6 \\
R(2+1)D [41] & No & 74.5 & 96.8 \\
SlowFast [8] & No & 79.3 & 96.8 \\
I3D+Flow [2] & Yes & 80.7 & 98.0 \\
R(2+1)D+Flow [41] & Yes & 78.7 & 97.3 \\
I3D + DMC-Net (I3D) [32] & Train & 77.8 & 96.5 \\
\hline SIFP (ours) & No & 72.3 & 94.0 \\
SIFP (ours) + SlowFast & No & 80.1 & 96.9 \\
\hline
\end{tabular}

generation for pseudo optical flows can be comparable for action recognition accuracy to those methods using optical flows directly.

It is proved that our unsupervised scheme for optical flow is reasonable and effective. Even without the optical flow supervision, we can still exceed most methods and achieve impressive results. Moreover, our approach also has the advantage of end-toend framework without multi-step training. More importantly, our method is time-saving and resource-saving compared with those schemes using optical flows. It usually should take a great deal of time and storage space to calculate optical flow features, especially considering the huge scales of current video action datasets.

Comparison with raw video based methods. The current typical streams of neural network models for raw video action recognition include motion representation [7, 28], 2D two-stream methods [11,33], and 3D convolution methods [2, 38, 41]. Although our SIFP method utilizes compressed videos for action recognition, it can achieve comparable or even better performance with some raw video based methods. In this section, we will compare our SIFP method with other raw video based methods. To further illustrate the effect of our model, we also combine our SIFP model with the SlowFast model [8] to recognize actions. In this setting, our SIFP model takes advantage of compressed videos and the SlowFast model relies on RGB frames.
Table 4: Ablation experiments on basic structure of our SIFP network (\%). SI indicates slow I pathway and FP means fast $P$ pathway. POF means the POF generators group, and RP Loss indicates the residual photometric loss.

\begin{tabular}{lccc}
\hline Method & Accumulated & HMDB51 & UCF101 \\
\hline SI & - & 69.0 & 91.7 \\
FP & Yes & 66.6 & 87.2 \\
SI+FP & No & 69.3 & 92.6 \\
SI+FP & Yes & 71.0 & 93.2 \\
SI+FP+POF & No & 70.5 & 93.0 \\
SI+FP+POF & Yes & 71.4 & 93.5 \\
SI+FP+POF+RP & Yes & 72.3 & 94.0 \\
\hline
\end{tabular}

Table 3 shows the action recognition accuracy comparison of our method with other approaches. In this table, the motion representation and 2D two-stream methods utilize raw RGB videos and optical flows for action recognition. Our SIFP only utilizes compressed videos for action recognition. However Table 3 shows that our SIFP method outperforms most comparison motion representation and 2D two-stream methods on the two datasets. This proves the advantage of our SIFP method. Compared with some 3D convolution methods for raw video action recognition, our SIFP achieves lower action recognition performance. However, the 3D convolution methods plus optical flows based on raw videos are time-consuming, while our method maintains a balance between accuracy and efficiency by avoiding the usage of optical flows and raw videos. When combining our SIFP with other raw video method such as SlowFast [8], the performance is remarkably improved. As shown in Table 3, the SIFP + SlowFast method outperforms most 3D convolution methods. Compared with SlowFast [8], the SIFP + SlowFast method improves the performance on both the datasets.

\subsection{Ablation Experiments}

Network structures. Our SIFP framework contains four key structure designs: slow I pathway (SI), fast P pathway (FP), pseudo optical flow (POF) generator group, and residual photometric loss (RP). In this section, we will show their effects with ablation studies. As shown in Table 4, we perform overall ablation experiments to validate these components. Additionally, because MV and Res have accumulated mode and non-accumulated modes, we test the performance of the two modes, respectively [50].

Table 4 shows that the slow I pathway (SI) can achieve better action recognition performance than the fast $\mathrm{P}$ pathway (FP), and their fusion further improves the performance. The basic POF generator group slightly improves the performance without the residual photometric loss (RP). With RP Loss, the accuracy is remarkably increased on the two datasets. This study demonstrates and validates the effect of each key structure components.

In addition, we observe that the use of accumulated MV and Res can improve the performance of our model. Furthermore, our RP loss is based on the assumption of using the accumulated MV and Res. Without them the pseudo optical flow of the current P-frame cannot correspond to the Res of the previous P-frames. 
Table 5: Ablation experiments on the structure of single POF generator (\%). "Basic generator" indicates the original optical flow generator without MV shortcut and RP Loss. "RP Loss" means the residual photometric loss. "MV+Res" means taking both MV and Res as inputs.

\begin{tabular}{lccc}
\hline Method & Input & HMDB51 & UCF101 \\
\hline Basic generator & MV & 69.4 & 92.4 \\
Basic generator & MV+Res & 71.2 & 92.7 \\
+MV shortcut & MV+Res & 71.4 & 93.5 \\
+MV shortcut+RP Loss & MV+Res & 72.3 & 94.0 \\
\hline
\end{tabular}

Pseudo optical flow (POF) generator structures. The POF generator is designed to refine MV and contains parts of Res, MV shortcut, and RP Loss. In this experiment, we test the effects of these parts with ablation study.

As shown in Table 5, we test the basic generator without other components, and then add Res, MV shortcut, and RP loss step by step. It can be seen that each component plays positive roles in the generator. RP Loss and MV shortcut are helpful to produce the large improvement of performance.

Visualization. We visualize three types of pseudo optical flows in different ablation settings, including the pseudo optical flow with non-accumulated MV (POF No-Acc), the pseudo optical flow without RP loss (POF No-RP), and the final pseudo optical flow (POF). For comparison, we also visualize the accumulated motion vectors (MV) and residuals (Res). Fig. 4 shows the comparison of the visualization.

This figure shows that the original MV is rough, lumpy, and has noisy motions in the background that is independent of the human. The motions in the background are undesired for human action recognition, and the rough motions of the human can not clearly show the specific body movements. Our method seeks to filter out the noisy motions in the background and refine the body movements of the foreground human.

It can be noticed that all the three types of pseudo optical flow generators successfully smooth the original MV and generate finer pseudo optical flows. Among them, the final pseudo optical flow is the finest. It fits well with the edges of Res and the movements of the person's body are clear. As the background is the cleanest, the optical flow is concentrated on the moving foreground characters. The pseudo optical flow without RP loss can also restore the motion information. However, the photometry is not the same in different frames due to the missing constraints of the photometric loss, resulting in a smooth background with varying shades. This problem also exists in the pseudo optical flow with non-accumulated MV. Due to the lack of long-range information, this type of POF brings granular background noise, which means that noise information such as camera movement is not well filtered out, and the photometric uniformity between frames is not maintained.

\section{CONCLUSION}

In this paper, we propose a Slow-I-Fast-P (SIFP) architecture for compressed video action recognition. The proposed method achieves state-of-the-art performance on the challenging compressed video

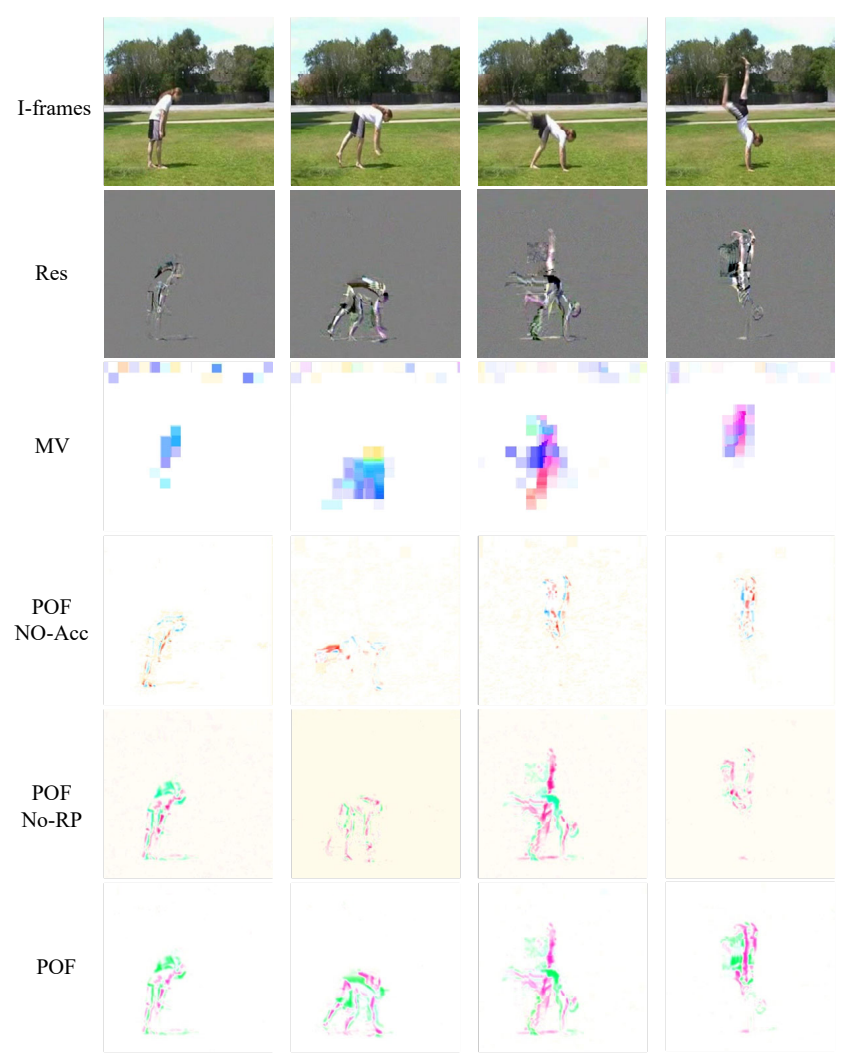

Figure 4: Visualization of the original input modalities(Iframe, Res, MV), and pseudo optical flow (POF) in three ablation settings, including the pseudo optical flow with nonaccumulated MV (POF No-Acc), the pseudo optical flow without RP loss (POF No-RP), and the final pseudo optical flow (POF).

benchmarks. We design an unsupervised estimation method and a new loss function to generate pseudo optical flows in compressed videos. The dependence on traditional resource-consuming optical flows is eliminated and the model is trained in an end-to-end manner. Extensive comparison and ablation experiments demonstrate the effectiveness and superiority of our proposed method.

In application scenarios, our method saves the cost of decoding and storing RGB frames and optical flow calculation, achieving the comparable accuracy as raw video action recognition. Therefore, our method has promising application prospects, such as online video understanding and security surveillance video processing.

\section{ACKNOWLEDGEMENT}

This research was supported by the National Key Research and Development Program of China (No. 2018AAA0102501) and National Natural Science Foundation of China (No. 61876149). The authors also thank Samsung R\&D Institute China Xi'an (SRCX) for the support. 


\section{REFERENCES}

[1] Daniel J Butler, Jonas Wulff, Garrett B Stanley, and Michael J Black. 2012. A naturalistic open source movie for optical flow evaluation. In European conference on computer vision. Springer, 611-625.

[2] Joao Carreira and Andrew Zisserman. 2017. Quo vadis, action recognition? a new model and the kinetics dataset. In proceedings of the IEEE Conference on Computer Vision and Pattern Recognition. 6299-6308.

[3] Yunpeng Chen, Yannis Kalantidis, Jianshu Li, Shuicheng Yan, and Jiashi Feng. 2018. Multi-fiber networks for video recognition. In Proceedings of the european conference on computer vision (ECCV). 352-367.

[4] Nieves Crasto, Philippe Weinzaepfel, Karteek Alahari, and Cordelia Schmid. 2019 Mars: Motion-augmented rgb stream for action recognition. In Proceedings of the IEEE conference on computer vision and pattern recognition. 7882-7891.

[5] AM Derrington and P Lennie. 1984. Spatial and temporal contrast sensitivities of neurones in lateral geniculate nucleus of macaque. The Journal of physiology 357, 1 (1984), 219-240.

[6] Alexey Dosovitskiy, Philipp Fischer, Eddy Ilg, Philip Hausser, Caner Hazirbas, Vladimir Golkov, Patrick Van Der Smagt, Daniel Cremers, and Thomas Brox. 2015. Flownet: Learning optical flow with convolutional networks. In Proceedings of the IEEE international conference on computer vision. 2758-2766.

[7] Lijie Fan, Wenbing Huang, Chuang Gan, Stefano Ermon, Boqing Gong, and Junzhou Huang. 2018. End-to-end learning of motion representation for video understanding. In Proceedings of the IEEE Conference on Computer Vision and Pattern Recognition. 6016-6025.

[8] Christoph Feichtenhofer, Haoqi Fan, Jitendra Malik, and Kaiming He. 2019. Slowfast networks for video recognition. In Proceedings of the IEEE International Conference on Computer Vision. 6202-6211.

[9] Christoph Feichtenhofer, Axel Pinz, and Richard Wildes. 2016. Spatiotemporal residual networks for video action recognition. In Advances in neural information processing systems. 3468-3476.

[10] Christoph Feichtenhofer, Axel Pinz, and Richard P Wildes. 2017. Spatiotemporal multiplier networks for video action recognition. In Proceedings of the IEEE conference on computer vision and pattern recognition. 4768-4777.

[11] Christoph Feichtenhofer, Axel Pinz, and Andrew Zisserman. 2016. Convolutional two-stream network fusion for video action recognition. In Proceedings of the IEEE conference on computer vision and pattern recognition. 1933-1941.

[12] Daniel J Felleman and DC Essen Van. 1991. Distributed hierarchical processing in the primate cerebral cortex. Cerebral cortex (New York, NY: 1991) 1, 1 (1991), $1-47$.

[13] Ruohan Gao, Bo Xiong, and Kristen Grauman. 2018. Im2flow: Motion hallucination from static images for action recognition. In Proceedings of the IEEE Conference on Computer Vision and Pattern Recognition. 5937-5947.

[14] Kaiming He, Xiangyu Zhang, Shaoqing Ren, and Jian Sun. 2016. Deep residual learning for image recognition. In Proceedings of the IEEE conference on computer vision and pattern recognition. $770-778$.

[15] Berthold KP Horn and Brian G Schunck. 1981. Determining optical flow. In Techniques and Applications of Image Understanding, Vol. 281. International Society for Optics and Photonics, 319-331.

[16] David H Hubel and Torsten N Wiesel. 1965. Receptive fields and functional architecture in two nonstriate visual areas (18 and 19) of the cat. Fournal of neurophysiology 28, 2 (1965), 229-289.

[17] Eddy Ilg, Nikolaus Mayer, Tonmoy Saikia, Margret Keuper, Alexey Dosovitskiy, and Thomas Brox. 2017. Flownet 2.0: Evolution of optical flow estimation with deep networks. In Proceedings of the IEEE conference on computer vision and pattern recognition. 2462-2470.

[18] J Yu Jason, Adam W Harley, and Konstantinos G Derpanis. 2016. Back to basics: Unsupervised learning of optical flow via brightness constancy and motion smoothness. In European Conference on Computer Vision. Springer, 3-10.

[19] Boyuan Jiang, MengMeng Wang, Weihao Gan, Wei Wu, and Junjie Yan. 2019. STM: SpatioTemporal and Motion Encoding for Action Recognition. In Proceedings of the IEEE International Conference on Computer Vision. 2000-2009.

[20] Chao Jing, Ping Wei, Hongbin Sun, and Nanning Zheng. 2020. Spatiotemporal neural networks for action recognition based on joint loss. Neural Computing and Applications 32 (2020), 4293-4302.

[21] Hildegard Kuehne, Hueihan Jhuang, Estíbaliz Garrote, Tomaso Poggio, and Thomas Serre. 2011. HMDB: a large video database for human motion recognition. In 2011 International Conference on Computer Vision. IEEE, 2556-2563.

[22] Didier Le Gall. 1991. MPEG: A video compression standard for multimedia applications. Commun. ACM 34, 4 (1991), 46-58.

[23] Myunggi Lee, Seungeui Lee, Sungjoon Son, Gyutae Park, and Nojun Kwak. 2018. Motion feature network: Fixed motion filter for action recognition. In Proceedings of the European Conference on Computer Vision (ECCV). 387-403.

[24] Margaret Livingstone and David Hubel. 1988. Segregation of form, color, movement, and depth: anatomy, physiology, and perception. Science 240, 4853 (1988) 740-749.

[25] Andrew L Maas, Awni Y Hannun, and Andrew Y Ng. 2013. Rectifier nonlinearities improve neural network acoustic models. In Proc. icml, Vol. 30. 3.
[26] Simon Meister, Junhwa Hur, and Stefan Roth. 2018. UnFlow: Unsupervised learning of optical flow with a bidirectional census loss. In Thirty-Second AAAI Conference on Artificial Intelligence.

[27] Moritz Menze, Christian Heipke, and Andreas Geiger. 2015. JOINT 3D ESTIMATION OF VEHICLES AND SCENE FLOW. ISPRS Annals of Photogrammetry, Remote Sensing \& Spatial Information Sciences 2 (2015).

[28] Joe Yue-Hei Ng, Jonghyun Choi, Jan Neumann, and Larry S Davis. 2018. Actionflownet: Learning motion representation for action recognition. In 2018 IEEE Winter Conference on Applications of Computer Vision (WACV). IEEE, 1616-1624.

[29] Zhaofan Qiu, Ting Yao, and Tao Mei. 2017. Learning spatio-temporal representation with pseudo-3d residual networks. In proceedings of the IEEE International Conference on Computer Vision. 5533-5541.

[30] Anurag Ranjan and Michael J Black. 2017. Optical flow estimation using a spatial pyramid network. In Proceedings of the IEEE Conference on Computer Vision and Pattern Recognition. 4161-4170.

[31] Laura Sevilla-Lara, Yiyi Liao, Fatma Güney, Varun Jampani, Andreas Geiger, and Michael J Black. 2018. On the integration of optical flow and action recognition. In German Conference on Pattern Recognition. Springer, 281-297.

[32] Zheng Shou, Xudong Lin, Yannis Kalantidis, Laura Sevilla-Lara, Marcus Rohrbach, Shih-Fu Chang, and Zhicheng Yan. 2019. Dmc-net: Generating discriminative motion cues for fast compressed video action recognition. In Proceedings of the IEEE Conference on Computer Vision and Pattern Recognition. 1268-1277.

[33] Karen Simonyan and Andrew Zisserman. 2014. Two-stream convolutional networks for action recognition in videos. In Advances in neural information processing systems. 568-576.

[34] Karen Simonyan and Andrew Zisserman. 2015. Two-stream convolutional networks for action recognition. In Proceedings of the Neural Information Processing Systems (NIPS).

[35] Khurram Soomro, Amir Roshan Zamir, and Mubarak Shah. 2012. UCF101: A dataset of 101 human actions classes from videos in the wild. arXiv preprint arXiv:1212.0402 (2012).

[36] Deqing Sun, Stefan Roth, JP Lewis, and Michael J Black. 2008. Learning optical flow. In European Conference on Computer Vision. Springer, 83-97.

[37] Deqing Sun, Xiaodong Yang, Ming-Yu Liu, and Jan Kautz. 2018. Pwc-net: Cnns for optical flow using pyramid, warping, and cost volume. In Proceedings of the IEEE Conference on Computer Vision and Pattern Recognition. 8934-8943.

[38] Du Tran, Lubomir Bourdev, Rob Fergus, Lorenzo Torresani, and Manohar Paluri. 2015. Learning spatiotemporal features with $3 \mathrm{~d}$ convolutional networks. In Proceedings of the IEEE international conference on computer vision. 4489-4497.

[39] Du Tran, Jamie Ray, Zheng Shou, Shih-Fu Chang, and Manohar Paluri. 2017. Convnet architecture search for spatiotemporal feature learning. arXiv preprint arXiv:1708.05038 (2017)

[40] Du Tran, Heng Wang, Lorenzo Torresani, and Matt Feiszli. 2019. Video classification with channel-separated convolutional networks. In Proceedings of the IEEE International Conference on Computer Vision. 5552-5561.

[41] Du Tran, Heng Wang, Lorenzo Torresani, Jamie Ray, Yann LeCun, and Manohar Paluri. 2018. A closer look at spatiotemporal convolutions for action recognition. In Proceedings of the IEEE conference on Computer Vision and Pattern Recognition. 6450-6459.

[42] David C Van Essen, Jack L Gallant, et al. 1994. Neural mechanisms of form and motion processing in the primate visual system. Neuron 13, 1 (1994), 1-10.

[43] Limin Wang, Wei Li, Wen Li, and Luc Van Gool. 2018. Appearance-and-relation networks for video classification. In Proceedings of the IEEE conference on computer vision and pattern recognition. 1430-1439.

[44] Limin Wang, Yuanjun Xiong, Zhe Wang, Yu Qiao, Dahua Lin, Xiaoou Tang, and Luc Van Gool. 2016. Temporal segment networks: Towards good practices for deep action recognition. In European conference on computer vision. Springer, $20-36$.

[45] Xiaolong Wang, Ross Girshick, Abhinav Gupta, and Kaiming He. 2018. Non-local neural networks. In Proceedings of the IEEE conference on computer vision and pattern recognition. $7794-7803$.

[46] Yang Wang, Yi Yang, Zhenheng Yang, Liang Zhao, Peng Wang, and Wei Xu. 2018. Occlusion aware unsupervised learning of optical flow. In Proceedings of the IEEE Conference on Computer Vision and Pattern Recognition. 4884-4893.

[47] Ping Wei, Hongbin Sun, and Nanning Zheng. 2019. Learning Composite Latent Structures for 3D Human Action Representation and Recognition. IEEE Transactions on Multimedia 21, 9 (2019), 2195-2208.

[48] Ping Wei, Yibiao Zhao, Nanning Zheng, and Song-Chun Zhu. 2017. Modeling 4D Human-Object Interactions for Joint Event Segmentation, Recognition, and Object Localization. IEEE Transactions on Pattern Analysis and Machine Intelligence (TPAMI) 39, 6 (2017), 1165-1179.

[49] Joachim Weickert, Andres Bruhn, and Christoph Schnörr. 2003. Lucas/Kanade meets Horn/Schunck: Combining local and global optic flow methods. (2003).

[50] Chao-Yuan Wu, Manzil Zaheer, Hexiang Hu, R Manmatha, Alexander J Smola, and Philipp Krähenbühl. 2018. Compressed video action recognition. In Proceedings of the IEEE Conference on Computer Vision and Pattern Recognition. 6026-6035.

[51] Saining Xie, Chen Sun, Jonathan Huang, Zhuowen Tu, and Kevin Murphy. 2018. Rethinking spatiotemporal feature learning: Speed-accuracy trade-offs in video 
classification. In Proceedings of the European Conference on Computer Vision (ECCV). 305-321.

[52] Christopher Zach, Thomas Pock, and Horst Bischof. 2007. A duality based approach for realtime TV-L 1 optical flow. In foint pattern recognition symposium. Springer, 214-223.

[53] Bowen Zhang, Limin Wang, Zhe Wang, Yu Qiao, and Hanli Wang. 2016. Real-time action recognition with enhanced motion vector CNNs. In Proceedings of the IEEE conference on computer vision and pattern recognition. 2718-2726.
[54] Bowen Zhang, Limin Wang, Zhe Wang, Yu Oiao, and Hanli Wang. 2018. Real-time action recognition with deeply transferred motion vector cnns. IEEE Transactions on Image Processing 27, 5 (2018), 2326-2339.

[55] Yi Zhu, Zhenzhong Lan, Shawn Newsam, and Alexander Hauptmann. 2018. Hidden two-stream convolutional networks for action recognition. In Asian Conference on Computer Vision. Springer, 363-378.

[56] Mohammadreza Zolfaghari, Kamaljeet Singh, and Thomas Brox. 2018. Eco: Efficient convolutional network for online video understanding. In Proceedings of the European Conference on Computer Vision (ECCV). 695-712. 